\title{
Shape Detection for Impeller Blades by Non-contact Coordinate Measuring Machine
}

\author{
Shimin Luo \\ School of Railway Tracks and Transportation, East China Jiaotong University, \\ Nanchang, P.R. China
}

\begin{abstract}
The impeller blades are very important and precise parts in turbine, but they can be worn and deformed easily. In this paper, we present a novel approach to detect the shape of the impeller blades. The proposed approach is to compare the designed 3D model of the impeller blades with the virtual 3D model of the tested part which is reconstructed by non-contact coordinate measuring machine. The non-contact coordinate measuring machine measures an object with optical triangulation, and then we can reconstruct a virtual 3D model for the part. In contrast to previous approaches which detect the parts with complex surface, the proposed shape detection approach is scatheless and reliable. Experimental results also show that our approach gets the shape of the tested part precisely and describes the deformed condition clearly.
\end{abstract}

Keywords: Impeller blade, Non-contact coordinate measuring machine, Shape detection, Optical triangulation, Complex surface, $3 \mathrm{D}$ reconstruction.

\section{Introduction}

An impeller is a rotor inside a tube or conduit used to increase the pressure and flow of a fluid. It is the rotating component of a centrifugal pump, usually made of iron, steel, bronze, brass, aluminum or plastic, which transfers energy to the fluid. As an important part of the impeller, the impeller blade directly contacts the fluid and mixes up the fluid so that it might be worn and deformed easily. But how to detect an impeller blade with complex surface is a difficult problem to be addressed.

In reverse engineering, the noncontact coordinate measuring approaches based on laser triangulation measurement principle use multiple views of cameras to get the depth information of the object [1-3]. The object to be tested can be measured rapidly and precisely without any damage. In recent years, this issues that how to measure object with complex surface becomes one of the hottest questions to be solved in computer vision fields and reverse engineering fields.

In this paper, we exploit one of the noncontact coordinate measuring approaches, that is binocular vision measurement, to reconstruct the virtual $3 \mathrm{D}$ model for the impeller blade. By comparing the designed 3D model with the virtual 3D model of the impeller blades, we can detect the shape change of the part to be tested. 
The rest of the paper is organized as follows. Section 2 describes how to get the 3D points cloud of the tested object by the binocular vision measurement. Reconstructing the 3D model of the impeller blades from the point cloud data is introduced in Section 3. Section 4 presents the comparing process. Finally, Section 5 gives the conclusion.

\section{Tested Part Digitalization}

The basic principle of binocular vision measurement is to use two cameras from different views to take pictures for the tested part, extract a series of central image pixels projected by the linear laser, and pair them in the corresponding images according to stereo matching, finally acquire the 3D coordinate values by pinhole model.

\subsection{Camera Calibration}

Camera calibration is a process that obtains the internal and external parameters of the CCD cameras, which aim at establishing a mathematical relationship between the $3 \mathrm{D}$ world coordinate system with the $2 \mathrm{D}$ image coordinate system. According to the pinhole model, we can transform from the 3D world coordinate system to the $2 \mathrm{D}$ image coordinate system by the following four steps [4]:

Transform from object space coordinate system to camera coordinate system;

The pinhole perspective transformation of transforming from camera coordinate system to $2 \mathrm{D}$ image coordinate system;

The distortion model that considered the first order distortion;

Transform from the actual image coordinate system to the image coordinate system.

Finally, we can map the spatial point $\mathrm{P}$ with $3 \mathrm{D}$ coordinate values $\mathrm{x}, \mathrm{y}, \mathrm{z}$ to the image pixel with 2D image coordinate values $\mathrm{u}, \mathrm{v}$ by Formula (1):

$$
s\left[\begin{array}{l}
u \\
v \\
1
\end{array}\right]=\left[\begin{array}{llll}
m_{11} & m_{12} & m_{13} & m_{14} \\
m_{21} & m_{22} & m_{23} & m_{24} \\
m_{31} & m_{32} & m_{33} & m_{34}
\end{array}\right]\left[\begin{array}{l}
x \\
y \\
z
\end{array}\right]
$$

where $\mathrm{x}, \mathrm{y}, \mathrm{z}$ is the coordinate values to be solved; $\mathrm{u}, \mathrm{v}$ is the image values; and $\mathrm{s}$, mi, $\mathrm{j}(\mathrm{i}=1,2,3 ; \mathrm{j}=1,2,3,4)$ is the camera parameters that can be got by the two-step camera calibration approach proposed by Tsai R Y [5-6].

\subsection{Laser Stripe Images Processing}

Noise reduction: The variability of the ambient light and the inside and outside noises produced by cameras lead to the laser stripe images contain a great deal of random noises, so the target features become dimness, therefore the quality of the 2D laser stripe images become worse, which affect the image segmentation, image thinning and 
image matching. Thus we need filtering algorithm to improve the quality of the images, Gaussian filtering algorithm and median filtering algorithm both can remove the noise points of the laser stripe images effetely, here one of them can be exploited in this situation.

Image segmentation: Image segmentation refers to the process that separate the target features from the background. In binocular vision measurement, the target parts of images are the laser stripe. Because the topology position of the laser strip is very important, and the gray information of the laser stripe is insignificant. So we can use image threshold segmentation algorithm to extract the image positions of the laser stripe. According to the intensity of the ambient light and light produced by the laser, the image threshold segmentation algorithm sets a threshold and describes the pixels that gray value is larger than the threshold with white pixel, also describes the pixels that gray value is smaller than the threshold with black pixel. After image segmentation, we can transform a gray image into a white-black image.

Laser stripe thinning: Since the projection width of the laser stripe on the images is about $1 \mathrm{~mm}$, the target laser stripe at each image row after noise reduction and image segmentation occupies more than one pixel, so we need thin the laser stripe. The aim of laser stripe thinning is to transform the projection of the laser stripe to single pixel at each row. The thinning algorithm must guarantee the geometry and topology property of the laser stripe remain unchanged. The gravity central algorithm is often used to get the single pixel laser stripe.

Stereo matching: In binocular vision measurement, in order to obtain the 3D coordinate values of the projection point on the laser stripe, we need to pair the pixel points on the two corresponding laser stripe in the left and right images which are taken by the two cameras at the same time, that is, match one pixel point on the laser stripe in the left image with one of the pixel points on the laser stripe in the right image. The matching algorithm must comply with the following principles: the unique constraint, the continuity constraint, the compatibility constraint, the epipolar constraint, and the order of consistency constraints. The epipolar constraint define the matching pixel points in the right image of a pixel point in left image must be on a specific lines called epipolar line. This constraint greatly reduces the number of pixel points to be verified, and the searching domains are transforming from $2 \mathrm{D}$ area to $1 \mathrm{D}$ area.

After the above digital image processing, we can extract the information of the left and right images to series of paired matching pixel points $(<\mathrm{uL}, \mathrm{vL}\rangle,<\mathrm{uR}, \mathrm{vR}>)$, which facilitate the subsequent calculations [7].

\subsection{Mathematical Model Establishment}

The Formula (2), and (3) are established after we push the coordinate values of the paired pixel points into the Formula (1) respectively and eliminate the unknown parameters.

Actually Formula (2) represents a line that is start with the left camera center to the pixel point ( $\mathrm{uL}, \mathrm{vL})$ on the left image, while Formula (3) represents a line that is start with the right camera center to the pixel point $(\mathrm{uR}, \mathrm{vR})$ on the right image. 


$$
\begin{gathered}
\left\{\begin{array}{l}
\left(u^{L} m_{31}^{L}-m_{11}^{L}\right) x+\left(u^{L} m_{32}^{L}-m_{12}^{L}\right) y+\left(u^{L} m_{33}^{L}-m_{13}^{L}\right) z=m_{14}^{L}-u^{L} m_{34}^{L} \\
\left(u^{L} m_{31}^{L}-m_{21}^{L}\right) x+\left(u^{L} m_{32}^{L}-m_{22}^{L}\right) y+\left(u^{L} m_{33}^{L}-m_{23}^{L}\right) z=m_{24}^{L}-u^{L} m_{34}^{L}
\end{array}\right. \\
\left\{\begin{array}{l}
\left(u^{R} m_{31}^{R}-m_{11}^{R}\right) x+\left(u^{R} m_{32}^{R}-m_{12}^{R}\right) y+\left(u^{R} m_{33}^{R}-m_{13}^{R}\right) z=m_{14}^{R}-u^{R} m_{34}^{R} \\
\left(u^{R} m_{31}^{R}-m_{21}^{R}\right) x+\left(u^{R} m_{32}^{R}-m_{22}^{R}\right) y+\left(u^{R} m_{33}^{R}-m_{23}^{R}\right) z=m_{24}^{R}-u^{R} m_{34}^{R}
\end{array}\right.
\end{gathered}
$$

In order to get the coordinate values $\mathrm{x}, \mathrm{y}, \mathrm{z}$ of the $3 \mathrm{D}$ point, we solve the unified equation of Formula (2) and (3). If the two lines intersect, the intersection point of the two lines is exploited to calculate the $3 \mathrm{D}$ point. If the two lines may not intersect, we define the approximate intersection as the point which is closest to the two lines. More precisely, whether two lines intersect or not, we define the approximate intersection as the point $P$ which minimizes the sum of the square distances to both lines [8], as shown in Figure 1.

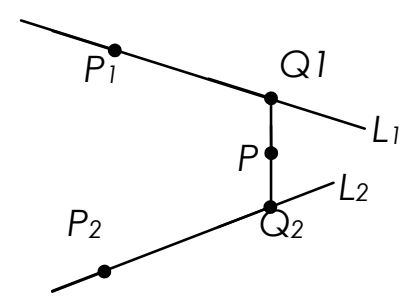

Fig. 1. The mathematical model of calculating the 3D points

\subsection{The Precision of Reconstruction}

To measure the reconstruction precision of the binocular vision measurement, we design an experiment to get the center coordinates of the four tangent circles.

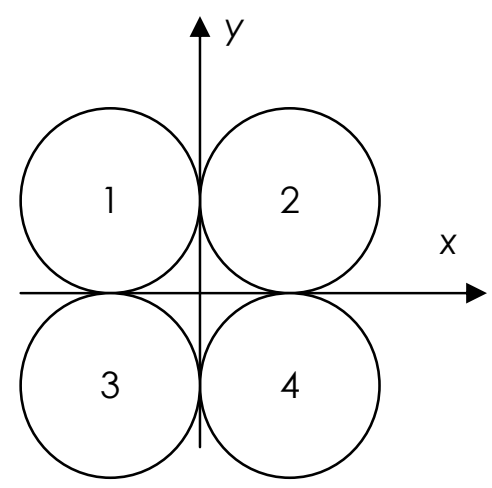

Fig. 2. The four circle centre is used to test the reconstructed precision 
We set the centre of the four circle centers as the world coordinate system centre, and make use of the above mathematical model to solve the coordinate values of the four circle centers. As the theoretical values of the coordinate values of the four circle centers is already known, we can get the measure precision by comparison the actual values with the theoretical values of the circle centers. The four circles are shown as Figure 2, and the measurement precision is given as Table I. From Table I, we conclude that the measurement accuracy is within $0.05 \mathrm{~mm}$; and this precision can satisfy with the need of industry.

Table 1. The reconstruction precision for the four circle centre

\begin{tabular}{|c|c|c|c|c|c|c|}
\hline & $\mathrm{x}$ & $\mathrm{y}$ & $\mathrm{z}$ & $\begin{array}{l}\text { Paired image } \\
\text { coordinate values }\end{array}$ & Distance & Distortion \\
\hline \multirow{2}{*}{1} & -30.0 & 30.0 & 0.0 & \multirow{2}{*}{$\begin{array}{l}(<123.3,132.0> \\
<184.4,111.4>)\end{array}$} & 42.426 & 0 \\
\hline & -30.023 & 30.003 & 0.040 & & 42.445 & 0.019 \\
\hline \multirow{2}{*}{2} & 30.0 & 30.0 & 0.0 & \multirow{2}{*}{$\begin{array}{l}(<121.5,194.3> \\
<184.3,179.2>)\end{array}$} & 42.426 & 0 \\
\hline & 29.966 & 30.009 & 0.067 & & 42.409 & -0.017 \\
\hline \multirow{2}{*}{3} & -30.0 & -30.0 & 0.0 & \multirow{2}{*}{$\begin{array}{l}(<367.3,131.5> \\
<377.3,373.6>)\end{array}$} & 42.426 & 0 \\
\hline & -30.017 & -30.004 & -0.124 & & 42.442 & 0.016 \\
\hline \multirow{2}{*}{4} & 30.0 & -30.0 & 0 & \multirow{2}{*}{$\begin{array}{l}(<366.0,197.8> \\
<437.0,117.9>)\end{array}$} & 42.426 & 0 \\
\hline & 30.001 & -30.017 & 0.042 & & 42.439 & 0.013 \\
\hline
\end{tabular}

\section{The Cloud Data Post-processing}

We get a lot of point cloud after digitizing the tested part of the impeller blades with the binocular vision measurement described in the previous section, which is a dense collection of surface samples of the impeller blades. We can deal the point cloud of the impeller blades with a dot-curve-surface-based surface reconstructed approach in order to get a visual CAD model [9-10]. First, we can obtain a full outline of the tested part through eliminating point cloud noise, merging the point cloud from different views. Secondarily, by sampling the point cloud to a certain percentage and cutting the section cross the cloud point, we can get a series of 3D-spline curse. After smoothing the 3D-spline curse, we make use of them to regenerate a satisfactory watertight surface by blending the surfaces. Finally, we materialize the surface model of the impeller blades and get the visual 3D model for the tested impeller blades. Notice that the above steps may have to do repeatedly. If we do not satisfied with the final reconstructed model, we must return to the previous step and redo it. The point cloud, curse model, and 3D model of the impeller blades are shown as Figure 3, 4, 5 respectively. 


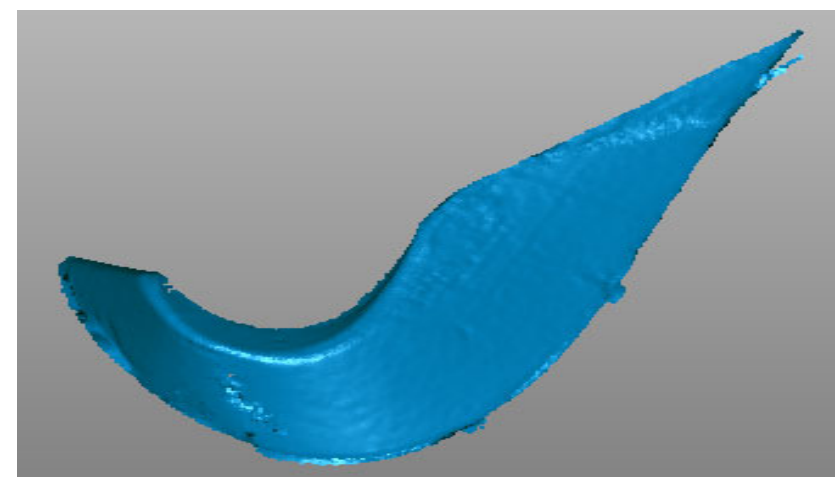

Fig. 3. The point cloud for the impeller blades to be tested

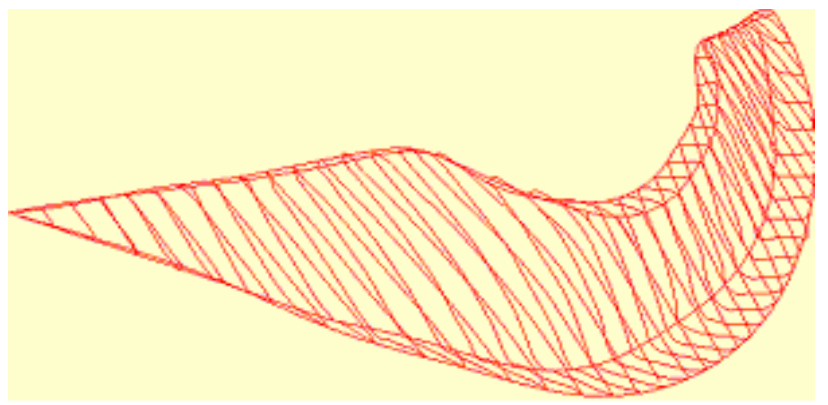

Fig. 4. The 3D-spline curse of the impeller blades to be tested

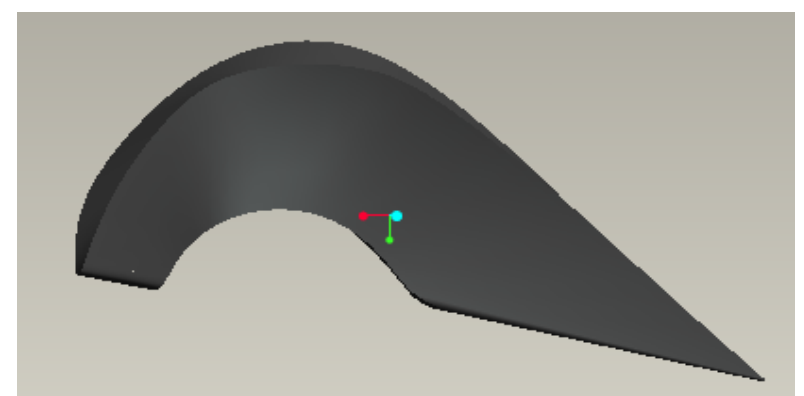

Fig. 5. The visual CAD model for impeller blades to be tested

\section{Comparisons}

After the 3D visual model of the tested part are reconstructed, we compare the 3D visual model with the designed 3D model that is already exist at the stage of part designing. 
The PRO/ENGINEERING commercial software can be exploited to compare the two 3D model of the part to be tested. Finally, we can get the surface deformation condition for the impeller blades, as shown in Figure 6, which describe different degrees of surface deformation with different colors.

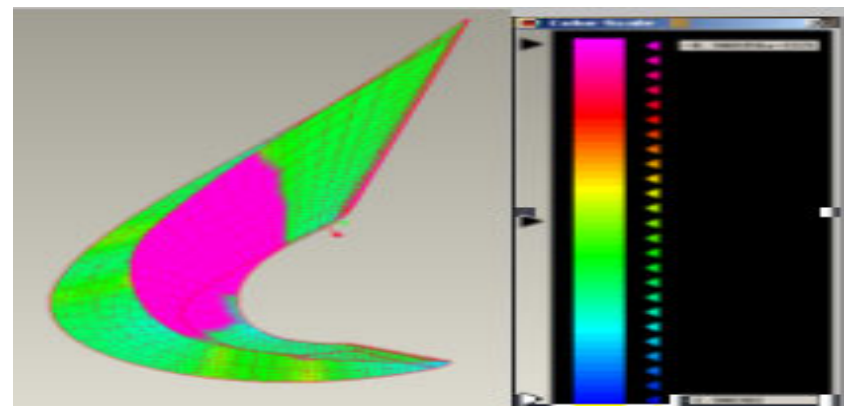

Fig. 6. The surface deformation condition for the impeller blades

\section{Conclusions}

In this paper, we propose a novel approach to detect the shape deformation condition for the impeller blades by comparing the designed 3D model with the virtual 3D model of the tested part that is reconstructed by non-contact coordinate measuring machine. In contrast to previous approaches which detect the parts with complex surface, the experimental results show our approach is scatheless and reliable.

\section{References}

1. Snavely, N., Seitz, S.M., Szeliski, R., Photo Tourism: Exploring image collections in 3D. ACM Transactions on Graphics (Proceedings of SIGGRAPH 2006) (2006)

2. Bradski, G., Kaehler, A.: Learning OpenCV: Computer Vision with the OpenCV Library, p. 32. O'Reilly Media, Inc., Sebastopol (2008)

3. Atcheson, B., Ihrke, I., Heidrich, W., Tevs, A., Bradley, D., Magnor, M., Seidel, H.-P.: Time-resolved 3d capture of non-stationary gas flows. ACM Transactions on Graphics (Proc.SIGGRAPH Asia) 27(5), article 132. 78 (2008)

4. Hirsch, M., Lanman, D., Holtzman, H., Raskar, R.: BiDi Screen: A Thin, Depth-Sensing LCD for 3D Interaction using Light Fields. In: ACM Transactions on Graphics (SIGGRAPH Asia 2009), Yokohama, Japan (December 2009)

5. Tsai, R.Y.: A Versatile Camera Calibration Technique for High-Accuracy 3D MachineVision Metrology Using Off-the-Shelf TV Cameras and Lenses. IEEE Journal of Robotics and Automation RA-3(4) (August 1987)

6. Zhang, Z.: Flexible camera calibration by viewing a plane from unknown orientations. In: International Conference on Computer Vision (ICCV), p. 40 (1999) 
7. Luo, S.-m., Li, M.-x.: Research on How to Get Object's 3D Coordinate on Two CCD Camera Measure System. Computer Engineering and Design 27, 3622-3624 (2006) (in Chinese)

8. Shapiro, L.G., Stockman, G.C.: Computer Vision. Prentice Hall, Englewood Cliffs (2001)

9. Radeva, P., Toledo, R., Von Land, C., Villanueva, J.: 3-D Vessel Reconstruction from Biplane Angiograms Using Snakes. Comput. Cardiol. 25, 773-776 (1998)

10. Cañero, C., Radeva, P., Toledo, R., Villanueva, J., Mauri, J.: 3-D Curve Reconstruction by Biplane Snakes. In: Proc. ICPR, vol. 4, pp. 563-566 (2000) 